\title{
Assessing the accuracy of intracameral phenylephrine preparation in cataract surgery
}

\author{
Stuart Guthrie ${ }^{1} \cdot$ Thomas Jensen $\mathbb{D}^{2} \cdot$ Richard C. Hartley ${ }^{2} \cdot$ Kanna Ramaesh ${ }^{1} \cdot$ David Lockington $\mathbb{D}^{1}$
}

Received: 3 February 2018 / Revised: 24 April 2018 / Accepted: 3 May 2018 / Published online: 15 June 2018

(C) The Royal College of Ophthalmologists 2018

\begin{abstract}
Purpose Unpreserved phenylephrine is often used as an off-licence intracameral surgical adjunct during cataract surgery to assist with pupil dilation and/or stabilise the iris in floppy iris syndrome. It can be delivered as a neat $0.2 \mathrm{ml}$ bolus of either 2.5 or $10 \%$ strength, or in a range of ad-hoc dilutions. We wished to assess the accuracy of intracameral phenylephrine preparation in clinical practice.

Methods Phenylephrine $0.2 \mathrm{ml}$ was analysed both neat (2.5 and 10\%) and in diluted form (ratio of 1:1 and 1:3). Samples were analysed using the validated spectrophotometric method.

Results A total of 36 samples were analysed. The standard curve showed linearity for phenylephrine $\left(R^{2}=0.99\right)$. Wide variability was observed across all dilution groups. There was evidence of significant differences in the percentage deviations from intended results between dilutions $(p<0.001)$. Mean percentage deviation for 1:3 dilution was significantly greater than neat $(p=0.003)$ and $1: 1$ dilution $(p=0.001)$. There was no evidence of a significant difference between $1: 1$ and neat $(p=0.827)$.

Conclusions Current ad-hoc dilution methods used to prepare intracameral phenylephrine are inaccurate and highly variable. Small volume $1 \mathrm{ml}$ syringes should not be used for mixing or dilution of drug. Commercial intracameral phenylephrine products would address dosage concerns and could improve surgical outcomes in cases of poor pupil dilation and/or floppy iris syndrome.
\end{abstract}

\section{Introduction}

The intracameral route is increasingly being utilised for drug delivery during cataract surgery [1-4]. Such medical adjuncts to surgery have been shown to reduce surgical complications and improve patient comfort [5-7]. Intracameral phenylephrine has been shown in numerous studies to be safe and effective with respect to intra-operative pupil dilatation and also in the management of floppy iris syndrome [1-10]. Some studies even suggest that intracameral phenylephrine is preferable to mechanical dilation devices

David Lockington

davidlockington@hotmail.com

1 Tennent Institute of Ophthalmology, Gartnavel General Hospital, 1053 Great Western Road, Glasgow G12 0YN, UK

2 WestCHEM School of Chemistry, University of Glasgow, Glasgow G12 8QQ, UK during phacoemulsification, in terms of ease of use, reduced surgical time and lower financial costs [11].

Intracameral phenylephrine has a predictable positive action on pupil dilation, with a moderate mydriatic effect demonstrated in concentrations ranging from $0.15 \mathrm{mg} / \mathrm{ml}$ to $5.0 \mathrm{mg} / \mathrm{ml}$ (approximately $4.3 \mathrm{~mm}$ diameter), and a nonlinear significant increase in pupil size at higher concentrations (mean pupil size $5.80 \mathrm{~mm} \pm 0.79$ (SD) for $15.0 \mathrm{mg} / \mathrm{mL}(1.5 \%)$ and $6.65 \mathrm{~mm} \pm 0.57$ for $30.0 \mathrm{mg} / \mathrm{mL}$ (3.0\%)) [12]. This phenomenon is due to the fact that phenylephrine may bind and stimulate receptors other than the $\alpha(1)$-receptor at the higher concentrations.

However, it has been our clinical observation that the degree of pupil dilatation varies considerably between patients and can be less predictable than the literature suggests. It is common surgical practice in the United Kingdom that phenylephrine is delivered as a neat $0.2 \mathrm{ml}$ intracameral bolus of either 2.5 or $10 \%$ strength (derived from preservative-free Minims (Bausch \& Lomb)) via a $1 \mathrm{ml}$ syringe, or following a range of ad-hoc dilutions, designed to reduce the exposure to associated excipients. These 
Table 1 Table showing mean descriptive results, the standard deviations (SD), standard error of the mean (SE Mean) and 95\% confidence intervals (CI) across a variety of dilutions

\begin{tabular}{lllllll}
\hline Variable & $\begin{array}{l}\text { Intended result } \\
(\mathrm{mg} / \mathrm{ml})\end{array}$ & $\begin{array}{l}\text { Number of } \\
\text { samples }\end{array}$ & Mean & SD & SE Mean & 95\% CI \\
\hline $10 \%$ neat & $(100)$ & 6 & 65.15 & 21.74 & 8.88 & $(42.33,87.97)$ \\
$10 \% 1: 1$ & $(50)$ & 6 & 61.52 & 20.77 & 8.48 & $(39.72,83.32)$ \\
$10 \% 1: 3$ & $(25)$ & 6 & 37.4 & 31.1 & 12.7 & $(4.7,70.0)$ \\
$2.5 \%$ neat & $(25)$ & 6 & 17.90 & 8.51 & 3.48 & $(8.97,26.83)$ \\
$2.5 \% 1: 1$ & $(12.5)$ & 6 & 11.667 & 2.374 & 0.969 & $(9.176,14.158)$ \\
$2.5 \% 1: 3$ & $(6.25)$ & 6 & 2.217 & 1.992 & 0.813 & $(0.126,4.308)$ \\
\hline
\end{tabular}

The intended result is in brackets for each dilution excipients include sodium metabisulphite, disodium edetate and purified water.

Previous studies have highlighted the potential for a high degree in variation of drug concentration when using small volume $1 \mathrm{ml}$ syringes for drug formulation, and have cautioned against this practice [13-19]. Similar concerns have been previously raised with respect to the preparation of intracameral and intravitreal antibiotics [13, 20,21].

As previously mentioned, there are a range of surgeon preferences for diluting phenylephrine for intracameral use, but these ad-hoc methods are without a clear evidence base. Additionally, such protocols have not been assessed to evaluate dosage accuracy in the clinical environment. One possible reason for the unpredictable response to intracameral phenylephrine could be that the surgeon is unintentionally administering an inaccurately low dosage. We therefore wished to assess the dosage accuracy of intracameral phenylephrine preparation in clinical practice.

\section{Methods}

To replicate the clinical scenario of phenylephrine use in the theatre environment, $1 \mathrm{ml}$ syringes (Becton Dickinson, Plastipak), $18 \mathrm{G} \times 1$ 1/2in blunt fill needles (Becton Dickinson) and $27 \mathrm{G} \times 7 / 8$ in Rycroft cannulae (Beaver-Visitec International) were used to draw up and deliver the solutions for analysis. Minims Phenylephrine hydrochloride $2.5 \%$ (Bausch \& Lomb; $0.5 \mathrm{ml}$ vials containing $12.5 \mathrm{mg}$ of drug) and Minims Phenylephrine hydrochloride 10\% (Bausch \& Lomb; $0.5 \mathrm{ml}$ vials containing $50 \mathrm{mg}$ of drug) were used as the source of phenylephrine. Balanced salt solution (BSS; Alcon) was used for the dilution protocols. Hydrochloric acid $(\mathrm{HCl}) 0.3 \%$ was additionally used for further necessary dilution, and to prevent precipitation of phenylephrine out of the solution which would result in inaccuracies in concentration measurement.

To accurately determine the concentration of the samples used in clinical practice, analysis was carried out using the spectrophotometric method [22]. This validated technique measures how much a chemical absorbs light by measuring the intensity of light as the beam passes through the sample solution. The degree of absorption measured can then be directly correlated to the concentration of the drug, in the form of a standard curve. The relationship between absorbance and concentration must be linear if the standard curve is to be used to determine the concentration of the unknown samples.

Phenylephrine absorption spectra range from 230 to 300 $\mathrm{nm}$ with a peak absorption of $273 \mathrm{~nm}$ [22]. Samples were therefore analysed using a spectrometer (model: Lambda 25 UV/VIS spectrometer, PerkinElmer) at an absorbance wavelength of $273 \mathrm{~nm}$. It was determined after a series of dilutions of phenylephrine that linearity in the standard curve was achieved with a dilution factor of 77 times. This factor of dilution was therefore applied to all the samples analysed and each sample was repeated 6 times. The sample's absorbance was then correlated against the standard curve to give the concentration of a $0.2 \mathrm{ml}$ solution.

The dilutions and analysis were carried out by an ophthalmology Specialty Registrar (SG) and a chemistry $\mathrm{PhD}$ student (TJ), in controlled conditions in the School of Chemistry, University of Glasgow, UK.

\section{Results}

The control standard curve for phenylephrine showed linearity with an $R^{2}=0.99$. As a result of this, the expected clinical concentration for $2.5 \%$ minims phenylephrine as a neat bolus would be $25 \mathrm{mg} / \mathrm{ml}, 12.5 \mathrm{mg} / \mathrm{ml}$ for the $1: 1$ dilution, and $6.25 \mathrm{mg} / \mathrm{ml}$ for the $1: 3$ dilution. The expected concentration for $10 \%$ minims phenylephrine as a neat bolus would be $100 \mathrm{mg} / \mathrm{ml}, 50 \mathrm{mg} / \mathrm{ml}$ for the $1: 1$ dilution, and $25 \mathrm{mg} / \mathrm{ml}$ for the $1: 3$ dilution.

A total of 6 samples for each of the 3 clinical dilution scenarios were analysed, for both the 2.5 and $10 \%$ phenylephrine minims, giving an overall total of 36 results. [See Table 1 for mean descriptive results, SDs, standard error of the mean and $95 \%$ confidence intervals]

To summarise the results using the $2.5 \%$ minims phenylephrine: for the neat undiluted bolus, the mean result was 
Table 2 Table showing mean percentage deviation from intended results

\begin{tabular}{|c|c|c|c|c|c|c|c|c|c|}
\hline Variable & Conc $(\%)$ & $N$ & Mean & SD & Minimum & Q1 & Median & Q3 & Maximum \\
\hline \multicolumn{10}{|c|}{ Descriptive results for neat bolus (no dilution) } \\
\hline \multirow[t]{2}{*}{ \% Deviation } & 10 & 6 & 33.20 & 23.37 & 10.70 & 13.78 & 24.30 & 62.33 & 63.00 \\
\hline & 2.5 & 6 & 35.1 & 25.5 & 6.0 & 9.6 & 36.6 & 59.3 & 62.0 \\
\hline \multicolumn{10}{|c|}{ Descriptive results for 1:1 ratio dilution } \\
\hline \multirow[t]{2}{*}{$\%$ Deviation } & 10 & 6 & 37.2 & 26.4 & 5.0 & 6.3 & 43.7 & 58.9 & 68.6 \\
\hline & 2.5 & 6 & 13.87 & 13.54 & 0.00 & 7.20 & 10.80 & 19.00 & 40.00 \\
\hline \multicolumn{10}{|c|}{ Descriptive results for 1:3 ratio dilution } \\
\hline \multirow[t]{2}{*}{$\%$ Deviation } & 10 & 6 & 105.8 & 68.8 & 18.8 & 44.0 & 95.6 & 183.1 & 192.4 \\
\hline & 2.5 & 6 & 68.4 & 29.1 & 26.4 & 43.2 & 67.6 & 100.0 & 100.0 \\
\hline
\end{tabular}

$17.9 \mathrm{mg} / \mathrm{ml}$ (range $9.5-30$, standard deviation (SD) 8.51). For the $1: 1$ dilution, the mean result was $11.67 \mathrm{mg} / \mathrm{ml}$ (range 7.5-14, SD 2.37). For the 1:3 dilution, the mean result was $2.21 \mathrm{mg} / \mathrm{ml}$ (range $0-4.7$, SD 1.99).

To summarise the results using the $10 \%$ minims phenylephrine: for the neat undiluted bolus, the mean result was $65.15 \mathrm{mg} / \mathrm{ml}$ (range 37-85.2, SD 21.74). For the 1:1 dilution, the mean result was $61.52 \mathrm{mg} / \mathrm{ml}$ (range 32.1-84.3, SD 20.77). For the $1: 3$ dilution, the mean result was $37.4 \mathrm{mg} / \mathrm{ml}$ (range 0-73.1, SD 31.1).

There was wide variability observed in all the different dilution groups when the actual results were compared with the expected results. This was particularly seen with the 1:3 dilutions for both 2.5 and $10 \%$, as the $95 \%$ confidence intervals did not contain the target values. (See Table 2 and boxplot in Fig. 1)

There was evidence of significant differences in the percentage deviations from intended results between the different dilutions (ANOVA; $p<0.001$ ). However, there was no evidence of a significant difference between the mean percentage deviations between the 2.5 and $10 \%$ concentrations for the different dilutions $(p=0.108)$,

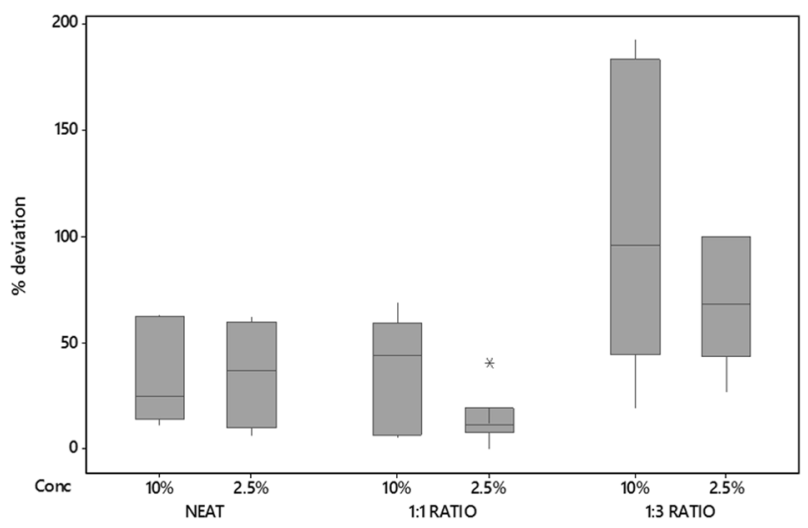

Fig. 1 Box plot demonstrating percentage deviation from expected results, showing variability across all 6 clinical scenarios, and greater variability associated with the 1:3 dilution protocols suggesting that the intrinsic protocol was being assessed in our study, rather than the original phenylephrine source. This allowed us to combine the results for both 2.5 and $10 \%$ into 3 groups, and perform statistical analysis (Tukey pairwise comparisons). Mean percentage deviation for the 1:3 dilution was significantly greater than for the neat group $(p=0.003)$, and also when compared to the $1: 1$ dilution groups $(p=0.001)$. There was no evidence of a significant difference between 1:1 dilution group and neat group ( $p=$ $0.827)$.

\section{Discussion}

We have demonstrated that there is a huge range of variability in the actual results and the difference from intended (expected) results when attempting to dilute intracameral phenylephrine, as demonstrated by the large $95 \%$ confidence intervals. Indeed, the 1:3 dilution group did not even contain the target value in their $95 \%$ confidence intervals. Our study has shown that the ad-hoc dilution methods currently used to prepare intracameral phenylephrine from Minims are inaccurate and can result in significantly lower concentrations of drug being administered. The potential consequences of such lower inadequate dosages are poor pupillary dilatation and continuation of floppy iris syndrome which could contribute to poorer cataract surgery outcomes. Additionally, it is important for the surgeon to accurately deliver a known concentration of any intracameral drug to reduce the potential risk of toxicity to the corneal endothelium associated with higher doses. A surgeon may be tempted to inject more intracameral phenylephrine on observing an ineffective response, and could inadvertently deliver unknown doses which could have an accumulative toxic effect. Particular care must be taken with phenylephrine in this regard, as it has been shown to have the highest concentration of free radicals of any of the commonly administered intracameral drugs, and accumulative free radical concentrations may contribute 
towards subsequent Toxic Anterior Segment Syndrome (TASS) [23, 24].

Our study shows that mathematical accuracy of these low volume dilution protocols do not correspond with accuracy in the clinical environment. This is predominantly due to the use of small volume $(1 \mathrm{ml})$ syringes, which are known to be inaccurate for the preparation of medications and should not be used for mixing of solutions [13-19]. There are 3 factors related to small volume syringes which may have contributed towards our observed findings in our study, those being dead space, air bubbles and concentration gradients.

Firstly, 'dead space' within the syringe refers to the volume of a solution retained in the hub and needle when the plunger of the syringe is fully depressed. One study estimated this volume at $0.07 \mathrm{ml}$ in a conventional $1 \mathrm{ml}$ syringe [19]. This volume represents $35 \%$ of the total $0.2 \mathrm{ml}$ expected intracameral dosage, and has the consequence that only $0.13 \mathrm{ml}$ of potential drug volume would be administered when using an undiluted neat "bolus". For the diluted protocols, this dead space volume represents undiluted BSS, which would subsequently be injected along with some (unknown) diluted drug solution. Both scenarios would result in a reduced intracameral drug concentration. Secondly, large air bubbles easily form within $1 \mathrm{ml}$ syringes, especially when drawing up small volumes of fluid from vials which have air pockets within them. This again has consequences of inaccurate dosing. Lastly, inadequate dilution within the syringe, due to the small chamber size preventing mixing, can result in a concentration gradient forming with the weakest dilution most anterior in the syringe. This weak $0.2 \mathrm{ml}$ would then subsequently be injected into the anterior chamber. (See illustration in Fig. 2)

We have observed a wide range of unexpectedly variable concentrations of drug across all the dilution protocols. Rather than diluting the excipients and optimising the drug, this means the surgeon is actually delivering a random quantity of drug when following such protocols.

We were interested to note the wide variability and inaccuracy associated with the use of a 1:3 dilution technique. As previously mentioned, minims phenylephrine contains sodium metabisulphite and disodium edetate as excipients. In real life, these results suggest that the surgeon

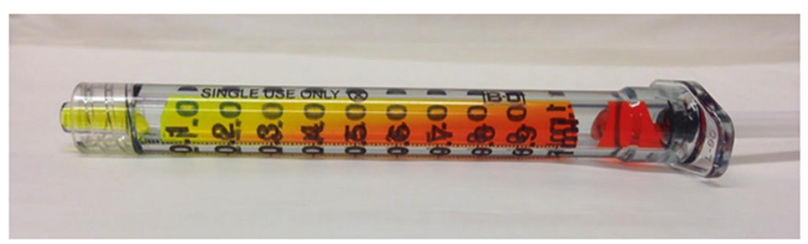

Fig. 2 Colour photograph showing the lack of mixing and dead space in a $1 \mathrm{ml}$ syringe, illustrated by drawing up a minim of Fluorescein and $0.5 \mathrm{ml}$ of water for injection who has chosen a $10 \%$ source to maximise exposure to phenylephrine, yet wishes to minimise the exposure to excipients through 1:3 dilution in a small volume syringe, could inadvertently be either delivering the equivalent of a full concentration neat $10 \%$ bolus, or alternatively, an extremely diluted solution with neither significant levels of drug or excipients. This range of inaccuracy and variability is obviously unacceptable, but could it be harmful?

Animal studies have investigated exposure to nonpreserved bisulfite-free phenylephrine $1.5 \%$ and demonstrated efficacy and safety [25]. Another study evaluating endothelial cell morphology and viability in the rabbit cornea did not detect any deleterious effect following intracameral exposure to bisulfite-containing phenylephrine (up to 1\%) [26]. This would account for the relative safety of the current operating room practice of diluting preservative-free minims of phenylephrine for intracameral use [27].

There are justified concerns regarding the safety of using off-licence intracameral preparations and the potential for errors [13, 28]. These include local ocular risks such as endothelial damage from excipients or preservatives, and incorrect dosages from compounding pharmacies resulting in cases of TASS [13, 28]. Rare cardiovascular effects such as hypertension have been reported, though the direct causation of such systemic events have been questioned [2932]. There has been a recently published case series of TASS following cataract surgery due to inadvertent administration of intracameral lidocaine and $2.5 \%$ phenylephrine preserved with $10 \%$ benzalkonium chloride, where 2 patients ultimately required corneal transplantation [33].

Our study has demonstrated the need for commercially available unpreserved intracameral phenylephrine to be available to ensure accuracy of dosage. There are currently 2 commercially available combination products which contain phenylephrine and are licenced for use in cataract surgery-Mydrane (Laboratoires THEA, Clermont-Ferrand, France), and Omidria (Omeros, Seattle). One bolus intracameral dose of $0.2 \mathrm{ml}$ Mydrane solution contains $0.04 \mathrm{mg}$ of tropicamide, $0.62 \mathrm{mg}$ of phenylephrine hydrochloride and $2 \mathrm{mg}$ of lidocaine hydrochloride. Omidria contains phenylephrine $1.0 \%$ and ketorolac $0.3 \%$; $4 \mathrm{mls}$ of which is diluted in $500 \mathrm{~mL}$ of the ophthalmic irrigating solution [34]. Both these products have been shown to be safe and effective in cataract surgery, to dilate and maintain pupil size by preventing intraoperative miosis, and reducing postoperative ocular pain [1, 2, 5-7]. These products do not require any additional preparation prior to use, and so eliminate any potential for dosage errors. In light of our study results demonstrating dosage variability with ad-hoc dilution of phenylephrine for intracameral use, it would be prudent to use a pre-prepared, purpose-designed, commercial surgical adjunct, for both safety and efficacy. 


\section{Conclusion}

Our study is the first to evaluate intracameral phenylephrine preparation protocols for dosage accuracy. We have used a validated technique to measure the concentrations of the drug under laboratory conditions. Small volume $(1 \mathrm{ml})$ syringes are known to be inaccurate for the preparation of medications and should not be used for mixing of solutions. Our findings provide support to the clinical observation that the degree of drug effect can vary considerably between patients, due to the potentially low and variable concentration of phenylephrine delivered intracamerally. Having bespoke commercial intracameral phenylephrine products available would address this problem and could improve cataract surgical outcomes in the setting of poor pupil dilation and/or floppy iris syndrome.

\section{Summary}

\section{What was known before}

- Intracameral adjuncts such as phenylephrine are increasingly used to improve pupil dilatation and address floppy iris syndrome in cataract surgery.

- These off-licence agents are often delivered following ad-hoc dilutions to minimise endothelial toxicity, but the accuracy of this practice has not been investigated.

\section{What this study adds}

- Current ad-hoc dilution methods used to prepare intracameral phenylephrine are inaccurate and highly variable.

- Small volume $1 \mathrm{ml}$ syringes should not be used for mixing or dilution of drug.

- Using commercial intracameral phenylephrine products would address this problem and improve surgical outcomes in cases of poor pupil dilation and/or floppy iris syndrome.

Acknowledgements We would like to thank Dr David Young (University of Strathclyde) for his assistance with the statistics. TJ has received studentship funding from the Engineering and Physical Sciences Research Council.

\section{Compliance with ethical standards}

Conflict of Interest DL and KR have received speaker honoraria for educational meetings from Thea Pharmaceuticals, however, that company had no involvement in this project. The remaining authors declare that they have no conflict of interest.

\section{References}

1. Lay Suan AL, Hamzah JC, Ken TS, Mansurali VN. Intracameral mydriatics versus topical mydriatics in pupil dilation for phacoemulsification cataract surgery. J Cataract Refract Surg. 2017;43:1031-5.

2. Behndig A, Korobelnik JF. Mydriatic insert and intracameral injections compared with mydriatic eyedrops in cataract surgery: controlled studies. J Cataract Refract Surg. 2015;41:1503-19.

3. Lundberg B, Behndig A. Intracameral mydriatics in phacoemulsification cataract surgery - a 6-year follow-up. Acta Ophthalmol. 2013;91:243-6.

4. Mori Y, Miyai T, Kagaya F, Nagai N, Osakabe Y, Miyata K, Amano S. Intraoperative mydriasis by intracameral injection of mydriatic eye drops: in vivo efficacy and in vitro safety studies. Clin Exp Ophthalmol. 2011;39:456-61.

5. Donnenfeld ED, Whitaker JS, Jackson MA, Wittpenn J. Intracameral ketorolac and phenylephrine effect on intraoperative pupil diameter and postoperative pain in cataract surgery. J Cataract Refract Surg. 2017;43:597-605.

6. Hovanesian JA, Sheppard JD, Trattler WB, Gayton JL, Malhotra RP, Schaaf DT, Ng E, Dunn SH. Intracameral phenylephrine and ketorolac during cataract surgery to maintain intraoperative mydriasis and reduce postoperative ocular pain: Integrated results from 2 pivotal phase 3 studies. J Cataract Refract Surg. 2015;41:2060-8.

7. Labetoulle M, Findl O, Malecaze F, Alió J, Cochener B, Lobo C, Lazreg S, Hartani D, Colin J, Tassignon MJ, Behndig A, Intracameral Mydrane Study 2 Group. Evaluation of the efficacy and safety of a standardised intracameral combination of mydriatics and anaesthetics for cataract surgery. $\mathrm{Br} \mathrm{J}$ Ophthalmol. 2016;100:976-85.

8. Vazquez-Ferreiro P,Carrera-Hueso FJ,Barreiro-Rodriguez L,DiazRey M,Poquet-Jornet JE,Ramón-Barrios MA,Sanjuan-Cerveró R, Effectiveness of intracameral phenylephrine in achieving mydriasis and reducing complications during phacoemulsification: a systematic review and meta-analysis. J Ocul Pharmacol Ther. 2017;33:735-42.

9. Lorente R, de Rojas V, Vázquez de Parga P, Moreno C, Varela J, Landaluce ML, Méndez J, Lorente B. Intracameral phenylephrine $1.5 \%$ for prophylaxis against intraoperative floppy iris syndrome: prospective, randomized fellow eye study. Ophthalmology. 2012;119:2053-8.

10. Lundqvist $\mathrm{O}$, Koskela $\mathrm{T}$, Behndig A. A paired comparison of intracameral mydriatics in refractive lens exchange surgery. Acta Ophthalmol. 2014;92:482-5.

11. Bucci FA Jr, Michalek B, Fluet AT. Comparison of the frequency of use of a pupil expansion device with and without an intracameral phenylephrine and ketorolac injection $1 \% / 0.3 \%$ at the time of routine cataract surgery. Clin Ophthalmol. 2017;11:1039-43.

12. Behndig A, Lundberg B. Mydriatic response to different concentrations of intracameral phenylephrine in humans. J Cataract Refract Surg. 2010;36:1682-6.

13. Lockington D, Flowers H, Young D, Yorston D. Assessing the accuracy of intracameral antibiotic preparation for use in cataract surgery. J Cataract Refract Surg. 2010;36:286-9.

14. Hall GH, Thompson CJ, Palmer A. Danger of dead space in U100 insulin syringes. Br Med J (Clin Res Ed). 1984;288:284-5.

15. Arnott RD, Cameron MA, Stepanas TV, Cohen M. Insulin syringes: dangers of dead space. Med J Aust. 1982;2:39-40.

16. Corcoran JS, Yudkin JS. How inaccurate is insulin mixing? Patient variability and syringe dead space effect. Diabet Med. $1985 ; 2: 131-3$ 
17. Watanachai A, Suprasongsin C. Deadspace: a potential error in concentration of medication during dilutional process in neonates. J Med Assoc Thail. 2003;86:1128-32.

18. Macfie AG. Equipment deadspace and drug administration. Anaesthesia. 1990;45:145-7.

19. Bhambhani V, Beri RS, Puliyel JM. Inadvertent overdosing of neonates as a result of the dead space of the syringe hub and needle. Arch Dis Child Fetal Neonatal Ed. 2005;90:F444-5.

20. Lockington D, Flowers H, Young D, Yorston D. Ensuring accuracy of intravitreal antibiotics and the need for training. $\mathrm{Br} \mathrm{J}$ Ophthalmol. 2009;93:1126.

21. Narvaez J, Wessels IF, Mattheis JK, Beierle F. Intravitreal antibiotics: accuracy of dilution by pharmacists, ophthalmologists, and ophthalmic assistants, using three protocols. Ophthalmic Surg. 1992;23:265-8.

22. Kryvanych AV, Bevz NY, Georgiyants VA. Verification of the quantitative determination method for phenylephrine hydrochloride in solution for injections. Acta Chim Pharm Indica. 2014;4:1-6.

23. Lockington D, Macdonald EC, Young D, Stewart P, Caslake M, Ramaesh K. Presence of free radicals in intracameral agents commonly used during cataract surgery. $\mathrm{Br} \mathrm{J}$ Ophthalmol. 2010;94:1674-7.

24. Lockington D, Macdonald E, Gregory M, Stewart P, Caslake M, Ramaesh K. Presence of free radicals in commonly used ophthalmic preparations. Br J Ophthalmol. 2010;94:525-6.

25. Nuijts RMMA, Mencucci R, Viaud-Quentric K, Elena PP, Olmière $\mathrm{C}$, Behndig A. Ocular tolerance in rabbits after intracameral administration of a fixed combination of tropicamide, phenylephrine, and lidocaine with and without rinsing. J Cataract Refract Surg. 2017;43:673-9.
26. Hong JW, Park JH, Kim ES, Kim JY, Kim MJ, Tchah H. Effect of intracameral injection of bisulfite-containing phenylephrine on rabbit corneal endothelium. Cornea. 2015;34:460-3.

27. Teoh LS, Foo SW, Mansurali VN, Ang EL, Md Noh UK, Bastion MC. Evaluation of corneal endothelial cell loss after uncomplicated phacoemulsification cataract surgery with intracameral phenylephrine. Asia Pac J Ophthalmol (Phila). 2017;6: 318-25.

28. Mamalis N, Edelhauser HF, Dawson DG, Chew J, LeBoyer RM, Werner L. Toxic anterior segment syndrome. J Cataract Refract Surg. 2006;32:324-33.

29. Bekir OA, Toufeeq S, Woods E, Jabir M. Effect of intracameral phenylephrine on systemic blood pressure. Eye (Lond). 2014;28:1267-8.

30. Williams GS, Radwan M, Kadare S, Williams CP. The short to medium-term risks of intracameral phenylephrine. Middle East Afr J Ophthalmol. 2012;19:357-60.

31. Shams F, Jafari AA, Mansfield D. Cardiovascular hazard of intracameral phenylephrine. J Cataract Refract Surg. 2015;41:2021-2.

32. Myers WG. Safety of intracameral phenylephrine. J Cataract Refract Surg. 2016;42:944-5.

33. Bielory BP, Shariff A, Hussain RM, Bermudez-Magner JA, Dubovy SR, Donaldson KE. Toxic anterior segment syndrome: inadvertent administration of intracameral lidocaine $1 \%$ and phenylephrine $2.5 \%$ preserved with $10 \%$ benzalkonium chloride during cataract surgery. Cornea. 2017;36:621-4.

34. Lawuyi LE, Gurbaxani A. The clinical utility of new combination phenylephrine/ketorolac injection in cataract surgery. Clin Ophthalmol. 2015;9:1249-54. 